\title{
Los juegos deportivos de blanco móvil: Propuesta de categorización e implementación desde un enfoque comprensivo, inclusivo y creativo Themoving target games: Aproposal of categorization and implementation from an understanding, inclusive and creative approach
}

\author{
Antonio Méndez Giménez \\ Universidad de Oviedo
}

Resumen: En este trabajo se definen y analizan las actividades lúdicas de persecución más evolucionadas, es decir, las que contemplan el lanzamiento de móviles para dar caza a los jugadores fugados. Una vez revisada la controversia en torno a la adecuación de uno de los más claros exponentes de este grupo de juegos, el dodgeball, se argumenta la necesidad de modificar las reglas para asegurar su potencial educativo, y se reformula la propuesta de esta categoría de juegos deportivos, los juegos de blanco o diana móvil, susceptible de ser abordada desde un planteamiento comprensivo. Esta familia de juegos deportivos viene a complementar la taxonomía de Almond (1986), y su consideración a nivel curricular podría tener implicaciones en la transferencia de principios tácticos. Igualmente, el artículo analiza las características estructurales y funcionales de este grupo de juegos, y plantea su puesta en escena utilizando materiales autoconstruidos y otorgando al alumno la posibilidad de inventar sus propios juegos.

Palabra clave: Juegos. Iniciación deportiva. Análisis estructural y funcional. Equipamiento autoconstruido. Modelo comprensivo. Invención de juegos.

\begin{abstract}
In this paper the more evolved tag games are defined and analyzed, that is, those characterized for throwing a soft object in order to hunt running away players. After reviewing the controversy about the adequacy of one of the clearest examples of this group of games, dodgeball, we argue the need to modify the rules to ensure their educational potential, and we consider a proposal of a new games category which could be addressed from an understanding approach. This family of games comes to complement the Almond's taxonomy (1986) and its curricular consideration could lead to possible implications on the transfer of tactical principles. Similarly, the article analyzes the structural and functional characteristics of this group of games, and defends the use of an, easy to create, homemade equipment, reducing the risk of causing damage to the opponent, and giving students the opportunity to invent their own games.
\end{abstract}

Key words: Games. Sport initiation. Structural and functional analysis. Homemade equipment. Tactical model. Games invention.

\section{Introducción}

Desdeel principio de la vida animal, y mucho antes de que apareciese la especie humana, los predadores han acechado, perseguido, acorralado y dado caza a sus presas. Las crías de muchas especies animales han jugado a forcejear, perseguirse o derribarse, en definitiva, a prepararse para el requerimiento de la fase adulta. Con la aparición de especies más evolucionadas, esa persecución también se ha desarrollado, añadiendo el uso de herramientas diversas y el lanzamiento de objetos que prolongan las acciones del cuerpo para hacer más eficaz la captura. Tradicionalmente, los niños de muchas generaciones, equipados con pistolas de agua o arcos y flechas con ventosa, han jugado a indios y vaqueros emulando a los héroes televisivos y de la gran pantalla, o han reproducido escenas de guerra arrojándose bolas de nieve o piñas, por lo quenopodemos eludir el origen bélico de estos juegos. Con la sofisticación del juego se ha dado lugar a prácticas deportivas más o menos cuestionables, como el paintball, en donde dos bandos con estética militar y pistolas de bolas de pintura se enfrentan disparando sobre sus adversarios para eliminarlos o conquistar territorios. Otros deportes, como la caza, el tiro al plato o la pesca submarina, ponen el énfasis en el desarrollo de puntería haciaun objeto o animal que se desplaza. Trataremos de entrever cuales son las aportaciones de este grupo de juegos a la Educación Físicay señalar los límites de su implementación en la sociedad del siglo XXI, caracterizada por la excesiva violenta.

La emergencia del modelo comprensivo (Thorpe, Bunkery Almond, 1986), y su continuo desarrollo y evolución (Griffin y Butler, 2005; Griffin, Mitchell y Oslin, 1997; Mitchell, Oslin y Griffin, 2003) han supuesto un cambio de foco en la enseñanza de los juegos deportivos, de manera que, en primera instancia, se prioriza más el qué hacer y cuándo (táctica) en cada situación de juego, que el cómo hacerlo (habilidades técnicas), aunque sin olvidarse de éstas. Con esa inversión del orden de los elementos técnico-tácticos frente al enfoque tradicional se pretende incluir a todo el alumnado en prácticas lúdicas, divertidas,

Fecha recepción: 30-09-09 - Fecha envío revisores: 19-10-09 - Fecha de aceptación: 16-03-10 Correspondencia: Antonio Méndez Giménez

$\mathrm{C} /$ Aniceto Sela, $\mathrm{s} / \mathrm{n}$

33005. Oviedo. Asturias

E-mail: mendezantonio@uniovi.es motivantes, auténticas y adaptadas a sus posibilidades. Se trata, no obstante, de una propuesta metodológica en la que no tiene cabida la oferta de juegos deslavazados o inconexos, sino la estructuración progresiva de situaciones que vayan apoyándose unas en otras y creciendo en complejidad táctica.

\begin{tabular}{|c|c|c|}
\hline \multicolumn{3}{|c|}{ Tabla 1. Juegos deportivos y juegos tradicionales de diana móvil. } \\
\hline Juegos tradicionales infantiles & Juegos deportivos & $\begin{array}{c}\text { Juegos tradicionales } \\
\text { adultos }\end{array}$ \\
\hline Balón prisionero & & \\
Liebres y cazadores & Dodgeball (EEUU y & Canadá) \\
Tiro al blanco & Caza \\
¡Pies quietos! & Rock-it-ball (Inglaterra) & Tiro al plato \\
La caza & Paintball & Pesca submarina \\
Gaga ball (Israel) & Tagball (EEUU) & \\
Deweke (Botswana) & & \\
Filling the bottle (Zimbawe) & & \\
\hline
\end{tabular}

La taxonomía bajo la que se ha construido el modelo comprensivo de enseñanza recogey ordena las modalidades deportivas que se practican exclusivamente con el empleo de algún elemento móvil, y sugiere algunas implicaciones metodológicas (Méndez, 2003). Así, entre las categorías de juegos deportivos propuestas por Almond (1986) encontramos una gama de familias que se podrían ubicar en algún punto de complejidad táctica entre los juegos de blanco o diana (Méndez, 2006a) y los juegos de invasión (Méndez, 2003; 2006b), tal es el caso de los juegos de cancha dividida y muro (Méndez 2003; 2005), y los juegos de fildeo y carrera (Méndez, 2009a). Al no encajar en la concepción del término anglosajón games, quedaron excluidos losjuegos deportivos psicomotores

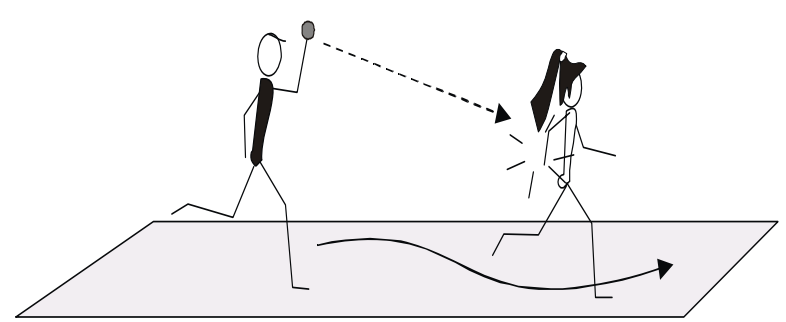

Fig. 1. Persecución por parejas. Tras ser cazada, la presa debe dar dos vueltas sobre sí misma, antes de recuperar la pelota y cambiar de roles. 


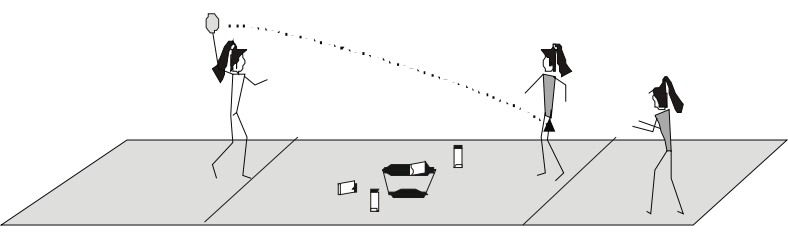

Fig. 2. Deweke (Botswana). El jugador central trata de introducir en un balde y con los pies, todas las latas dispersas por el suelo sin ser tocado por los disparos de los cazadores que lanzan desde los extremos. En la variante filling the bottle, el jugador central trata de llenar de arena una botella, en esta ocasión, empleando las manos.

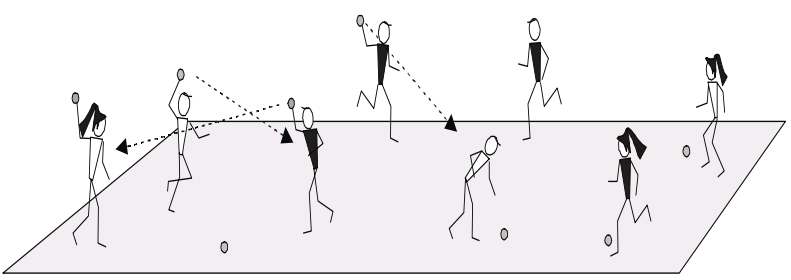

Fig. 3. Guerra de globos de agua, piñas, bolas de nieve...

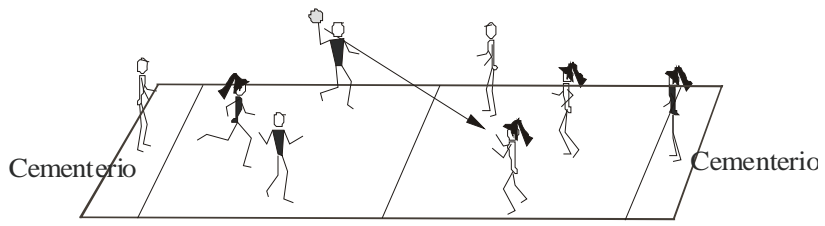

Fig. 4. Balón prisionero
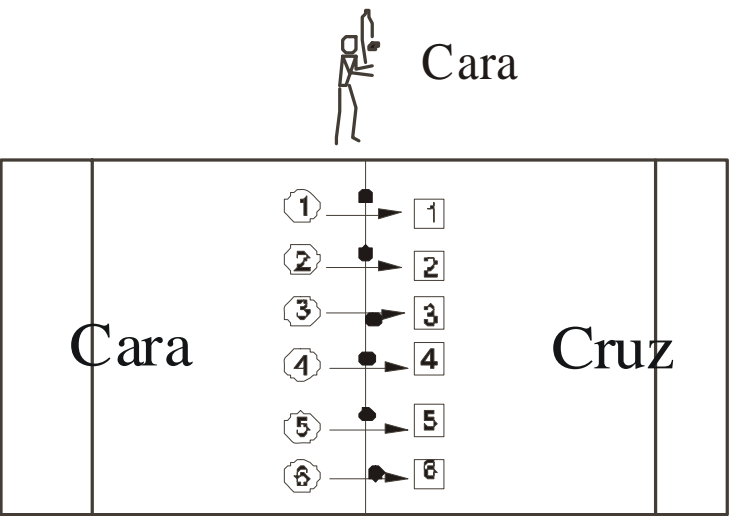

Fig. 5. Cara y cruz con balón. Si sale cara, los jugadores de la izquierda avanzan a por las pelotas de la línea central y tratan de cazar a los del otro bando antes de que sobrepasen la línea de fondo de su propio campo.

como las pruebas atléticas, la natación, la gimnasia y otras muchas modalidades sociomotoras, como la lucha, donde no se emplean objetos de intercambio. Sin embargo, esta clasificación tampoco contempló una categoría de juegos de blanco o diana móvil, ypensamos que es momento de replantear su posible inclusión y de sugerir su implementación metodológica a partir del modelo comprensivo, una vez justificado convenientemente su potencial didáctico.

A nivel conceptual, en este grupo de juegos determinados jugadores asumen el papel de cazadores y tienen como principal objetivo alcanzar, con la ayuda del móvil (generalmente, una pelota), el cuerpo de otros jugadores que ocupan el papel de liebres y que, a su vez, tratan de evitar ser alcanzados por los primeros (González, 1996; Hanrahan y Carlson, 2000). Puede jugarse en un espacio común o diferenciado y con roles estables o inestables. Entre otros ejemplos de juegos de blanco móvil institucionalizados encontramos el dodgeball, el rock-it-ball o el paintball. A ellos se pueden añadir otros muchos juegos practicados en Educación Física (balón prisionero, la caza, tiro al blanco, cara y cruz, cacería de leopardos o caza de la paloma)y juegos tradicionales infantiles, tanto de dentro como de fuera de nuestras fronteras (deweke, gaga ball, o filling the bottle), algunos de los cuales presentamos, brevemente, a continuación.

Tradicionalmente, los juegos de persecución (tag games) rudimentos de los juegos de diana móvil— han sido ampliamente utilizados en todas las etapas educativas como recursos motivadores en las clases de Educación Física y como estrategias efectivas para incrementar la actividad y la frecuencia cardiaca (Hanrahan y Carlson, 2000). De hecho, constituyen una forma eficaz de trabajar la resistencia orgánica a modo de cambios de ritmo y fartlek (Belka, 2006). Puesto que sus reglas son simples, no se precisan grandes explicaciones y, si se evitan las eliminaciones y se diseñan oportunamente, incentivarán la implicación de todos los alumnos. Además, muchos juegos depersecución se pueden transformar fácilmente en juegos de diana móvil empleando un móvil blando e incluyendo alguna regla que determine su manejo (cómo pasar, recibir o driblar). No obstante, la utilización esporádica de este tipo de juegos a lo largo del currículo, de manera inconexa con otras modalidades de la misma o distinta categoría, o de manera irreflexiva sobre los roles y principios generales que los gobiernan, resulta una praxis docente limitada que podría enriquecerse a partir de un planteamiento más comprensivo.

2. Los juegos de blancos humanos: una herramienta pedagógica controvertida

Varios autores han formulado críticas más o menos duras sobre algunos de los juegos que representan acciones de disparo hacia blancos humanos, bien, apelando a su cuestionamiento ético o, bien, argumentando que su propia naturaleza limita la cantidad de actividad física. Williams (1992, 1994) realizó su crítica ideando el Hall de la Vergüenza de Educación Física. Sus ataques al dodgeball y, por extensión, a los juegos de esta categoría, se centraron en los siguientes aspectos: la alta probabilidad de provocar situaciones de peligro, lesión o hacer daño; el excesivo énfasis en la eliminación de jugadores, el hecho de que su objetivo principal sea disparar hacia dianas humanas, y la inexistencia de deportes de referencia sobre los que poder edificar la práctica en la vida adulta. En su opinión, muchos juegos de persecución tienen una actividad física reducida, requieren pocas habilidades docentes, apenas promueven objetivos específicos del área y ponen a los estudiantes en situaciones embarazosas frente a sus compañeros.

Reglas del dodgeball

$\varnothing$ En cada equipo juegan 5 jugadores, otros 5 son suplentes.

Ø El campo, de 50 x 25 m, está dividido en 2 partes y cuenta con una línea de ataque situada en cada cancha a $3 \mathrm{~m}$ de la línea central. Los más pequeños suelen jugar en una cancha de voleibol, eliminando las líneas de ataque a tres metros.

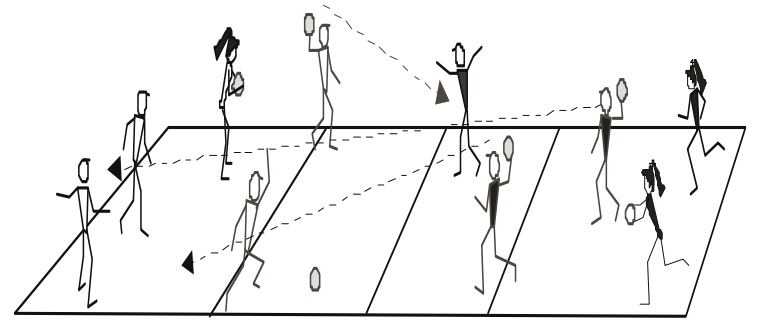

Fig. 6. Dodgeball

$\varnothing$ El objetivo es eliminar a los jugadores de equipo contrario lanzando una pelota directamente al cuerpo del rival y golpeándole por debajo de sus hombros.

$\varnothing$ Si se captura una pelota al vuelo, el jugador que la lanzó es eliminado y el equipo que la atrapó tiene derecho a incorporar a un jugador.

$\varnothing$ Los jugadores deben estar dentro de los límites del campo; pueden salir para recuperar la pelota pero han de regresar siempre por la línea de fondo. 
Ø Al iniciar el juego, las pelotas deben estar sobre la línea central del campo y los jugadores detrás de sus respectivas líneas de fondo. E árbitro hará sonar el silbato y los jugadores deben ir a buscar las pelotas.

$\varnothing$ Pierde el equipo que se quede sin jugadores en el campo. El juego consiste en 2 periodos de 5 minutos con un descanso de 2-5 minutos.

$\varnothing$ Si antes de concluir el tiempo se elimina a todos los jugadores de un equipo, vence el otro. Si termina la segunda parte, el equipo con más jugadores en campo, gana. En caso de que ambos bandos tengan la misma cantidad de jugadores vivos, se añadirá un tiempo extra de 1 minuto.

$\varnothing$ Cada equipo tiene derecho a un tiempo muerto de 30 segundos en cada periodo.

Ø Si todas las pelotas se encuentran en un lado del campo durante 5 segundos se comete falta y un jugador del equipo infractor deberá abandonar el terreno de juego.

Por su parte, Maurer (2006) cuestiona el dodgeball porque, en su opinión, no contribuye al desarrollo de ninguno de los estándares nacionales de la Educación Física. La dinámica de este juego aporta limitadas oportunidades de práctica, generalmente, no se aborda la enseñanza de la táctica y estrategia asociada al juego, y cuenta con escasa transferencia al ámbito extraescolar. Además, considera que promociona conductas agresivas y que aporta pocos beneficios para la salud, especialmente de los menos habilidosos, que no disponen de suficientes posibilidades de participación.

Jackson (2001) y McDonald (2005) revisaron los pros y contras del dodgeball y, por extensión, de los juegos de esquiva. Además de los argumentos ya mencionados, los detractores señalaron que este deporte es demasiado competitivo y sexista, puesto que los varones abusan de su fuerza para eliminar a los más débiles, y criticaron el uso de un equipamiento inadecuado o nombres violentos asociados al juego. Los defensores valoraban la sencillez, lo divertido que es jugarlo y el desarrollo de cualidades como los reflejos y la coordinación óculo-manual.

Otros autores (Belka, 2006; Deutsch, 2007; Méndez, 2001) sugieren que las acciones físico-motrices no son éticamente reprochables por sí mismas, sino que dependen de las reglas que las definan y del clima o contexto generado cuando se aplican. En cualquier caso, desde un planteamiento educativo es necesario modificar o transformar los juegos para dotarles de interés y dirigirlos a la consecución de los objetivos deseados a todos los niveles (motor, físico, táctico, valores), aprovechando para ello su propia naturaleza o esencia (Méndez, 2003). Además, la crítica de falta de conexión entre táctica y técnica parece ser más otro ejemplo de una mala praxis docente (incluso en la aplicación del modelo técnico hegemónico) que un motivo para el cuestionamiento de este deporte. Igualmente, la excesiva competitividad puede ser suavizada mediante regulaciones apropiadas que equilibren el potencial de los bandos para jugar en igualdad de condiciones o mediante la aplicación de estructuras de aprendizaje cooperativo (Casey, Dyson y Campbell, 2009).

No podemos cambiar el origen de estos juegos pero sí enfatizar los aspectos que más nos interesen para provocar los resultados deseados. En el último apartado daremos pautas para el diseño apropiado de juegos de diana móvil. Como argumentaremos a continuación, los juegos de blanco móvil cuentan con suficientes méritos para ser abordados en iniciación deportiva teniendo algunas ideas en mente como no considerar eliminaciones, priorizar la seguridad y tratar de aumentar la diversión de TODOS los participantes. Disparar una pelota blanda hacia el torso o las piernas de un oponente en un contexto lúdico y en igualdad de oportunidades no parece una actividad humillante ni vergonzosa y puede suponer un notable desafío para los jugadores. Al igual que no se cuestionan las actividades luctatorias o de forcejeo cuando se introduce al alumnado a deportes de combate o artes marciales, deberíamos ver estos juegos con perspectiva y en positivo, y valorar sus posibilidades para desarrollar la condición física, mejorar habilidades motrices, plantear problemas tácticos y abordar temas socio-afectivos.

\section{Justificación de la categoría y aplicación del modelo} comprensivo

Existe un antecedente de propuesta de clasificación de este grupo de juegos deportivos, se trata de la aportación de González (1996). En su análisis de los juegos deportivos desde un planteamiento comprensivo, la autora subdivide los juegos de blanco o diana en juegos de alcanzar blancos materiales y juegos de alcanzar blancos humanos. En nuestro caso, creemos más conveniente diferenciar entre los juegos de diana fija y los juegos de diana móvil, (ya sean blancos humanos o materiales los que se encuentran en movimiento), puesto que es esta característica, la de la movilidad del blanco, la que confiere una mayor dificultad tácticatécnica.

Creemos que en estos momentos disponemos de los suficientes argumentos para defender una categorización de estos juegos y de su sistematización educativa:

1.Existe un importante conjunto de juegos tradicionales infantiles que pertenecen a esta categoría tanto dentro (balón tiro, la caza, tiro al blanco, guerra de bolas de nieve...), como fuera de nuestras fronteras (deweke, filling the bottle, gagaball...)

2.Aunque en España todavía no se trata de modalidades muy conocidas, en otros países ya se han institucionalizado varios juegos deportivos (como el dodgeball en EEUU o el rock-it-ball en el Reino Unido), es decir, cuentan con federaciones que los promueven y que gestionan sus competiciones locales, regionales, naciones e internacionales. Además, su material ha sido patentado y comercializado. Por tanto, cumplen con las características necesarias para ser consideradas por el profesor Hernández Moreno (1994) como deportes (situación motriz reglada de carácter lúdico e institucionalizada).

3.Pese a las críticas presentadas, existen modalidades adultas institucionalizadas consideradas socialmente como deportes con gran arraigo, como la caza, el tiro al plato y recientemente el paintball.

4.Son juegos con gran riqueza táctica que, por tanto, requieren una implicación cognitiva y estratégica, además de demanda motriz, cuando se diseñan adecuadamente.

5.Además, enfatizan acciones táctico-técnicas comunes y transferibles a otras categorías deportivas. Por ejemplo, los cambios de ritmo y de dirección del jugador fugado y las fintas en los desplazamientos y lanzamientos del perseguidor son acciones intuitivamente transferibles a los movimientos de desmarque del atacante sin balón en los deportes de invasión (fútbol, baloncesto, hockey....). El remate hacia la manos del bloqueador para que el balón rebote y caiga fuera del campo de voleibol, la acción in extremis del jugador de baloncesto que lanza en el aire el balón para que toque al defensa antes de salir del terreno de juego, el pase a un compañero mejor situado en los juegos de invasión, la eliminación de corredores en los juegos de golpeo y fildeo, o la acción éticamente cuestionable de buscar el cuerpo del adversario en los deportes de cancha dividida, también son acciones compartidas.

6.En cuanto a la transferencia a la vida adulta, es importante contar con experiencias de esquiva de móviles o a interceptarlos, eso sí, suaves, que permitan desarrollar la habilidad de evitarlos. Encontramos varias aplicaciones prácticas de las habilidades que se desarrollan para la vida diaria, como desplazarse en medio de una masa de gente por la calle o centro comercial concurrido o conducir un coche en una gran ciudad. Hace poco, el mundo entero vio por televisión cómo el propio expresidente de los EEUU, George Bush, tuvo que hacer valer sus habilidades de esquiva para sortear un par de zapatos lanzados por un periodista iraquí durante una rueda de prensa.

7.Se trata de juegos deportivos simples de preparar y que despiertan la motivación del alumnado, seguramente por su flujo y movilidad. Asimismo, el equipamiento requerido se puede construir fácilmente con material reciclado.

8.Por último, los juegos de persecución y de blanco móvil pueden ser modificados para desarrollar el contenido curricular de manera integrada (Townsend, Mohr, Waronsky y Grana, 2006), así como las competencias básicas. La idea es incluir tareas o preguntas a los jugadores 
cazados relativas a las los contenidos de las áreas antes de reincorporarse aljuego.

Pasamos a ejemplificar una aplicación del modelo comprensivo (se va del cuándo y el qué al cómo) contemplando tres fases de enseñanza (Méndez, 2009b). Primero, se parte de un juego modificado para facilitar una experiencia gratificante al alumnado, después se trata de suscitar la conciencia táctica mediante tareas reflexivas y depurar la habilidad. Finalmente, se reintegra el patrón en el juego anterior o en otro más evolucionado buscando la transferencia. En esta propuesta, el objetivo es concienciar sobre la habilidad de esquiva y los cambios de dirección en defensa.

1.Juego modificado: Pelota al canguro. En un cuadro delimitado, dos jugadores situados en los extremos lanzan rodando una pelota ligera para impactar en los pies de un tercero (canguro) que se desplaza a saltos.

2.Conciencia táctica. ¿Qué haces para que no te den con la pelota? ¿Cómo puedes engañarles? ¿Qué tipo de desplazamientos realizas? ¿Cómo te desplazarías mejor para no agotarte?

Práctica de habilidad. Cambios de ritmo y dirección en carrera. Actividades de reflejos (agacharse, saltar, elevar piernas...)

3.Vuelta al juego. En esta ocasión, el perseguido se puede desplazar en carrera libremente esquivando el móvil. El lanzador que haga diana directa en las piernas del canguro, cambia su función con él.

\section{Características estructurales y funcionales}

Analizaremos con más detalle los elementos estructurales y funcionales de estos juegos:

1. Móviles. Generalmente se han empleado los balones deshinchados de otras modalidades deportivas, como los de fútbol o voleibol, o pelotas de rítmica o de floorball, no obstante, en la actualidad también existe la posibilidad de adquirir los comercializados y específicos (balones de dodgeball, rock-it-ball, goma espuma) o construirlos.

2. Implemento. Apenas en la modalidad de rock-it-ball se utiliza un stick con una cesta a cada lado, similar al empleado en intercrosse.

3. Meta. Lo constituye alguna zona corporal de los jugadores, y también diferentes objetos (balones medicinales, pelotas) que se desplazan.

4. Terreno de juego. González (1966) determina juegos realizados en un espacio común y juegos en espacio diferenciado. Obviamente, estos espacios pueden coincidir en un mismo juego dependiendo de las fases (por ejemplo, en rock-it-ball, se comienza en cancha dividida y tras apoderarse de las pelotas, se pasa a jugar en todo el campo). Por otro lado, en gaga ball se requiere un cercado del terreno de juego mediante paredes de un metro de altura aproximadamente y forma octogonal, contra el que rebota el balón.

5. Jugadores. Son juegos donde existe oposición y en ocasiones cooperación, por tanto, se juegan en equipos más o menos numerosos.

6. Tiempo. Solo los juegos institucionalizados determinan los tiempos de duración y el ritmo de juego.

7. Reglamento. Se trata de juegos con participación simultánea o alternativa de los roles de perseguidor y perseguido. Dichos roles pueden ser estables, inestables y ambivalentes.

8. Habilidades y técnicas deportivas. Entre las habilidades requeridas en los deportes de esta categoría destacan las referidas a la locomoción (marcha, carrera y salto), y las referidas al manejo del balón (lanzamiento, bote, recepción). Igualmente, se desarrollan las habilidades de esquiva, cambios de dirección y fintas.
9. Principios tácticos comunes. Los trabajos de Belka (1998; 2006) detallan los principios tácticos comunes a los juegos de persecución, entre otros, adoptar una posición equilibrada y lista para moverse en cualquier dirección, usar diferentes tipos de fintas tanto en ataque como en defensa, fintar cambiando la dirección y velocidad de movimiento de forma rápida e inesperada y tomar conciencia espacial de lo que ocurre alrededor. Para el autor, estos principios son transferibles a los deportes de invasión.

Por su parte, González (1996) enumera los principios tácticos de los juegos de de alcanzar blancos humanos. Para los cazadores (ataque) son los siguientes: orientarse y buscar la posición más adecuada, lanzar la pelota con precisión para alcanzar el blanco, decidir el tipo y forma de lanzamiento más idónea, pasar al compañero mejor situado, cerrar los espacios para rodear a las liebre y, facilitar la progresión del móvil. Para el rol de liebre (defensa); desplazarse rápidamente para alejarse del cazador, elegir la trayectoria de huída más conveniente, cambiar la dirección y el ritmo en la huída, organizar el espacio de acción y de juego para atrapar la pelota y, esquivar la pelota.

A lo comentado en los párrafos anteriores (además de los previstos para los juegos de persecución y los de diana móvil), habría que añadir, en ataque, otros principios que se dan en determinados juegos como hacerse con la posesión de un objeto o territorio o, en defensa: atrapar o interceptar la pelota, protegerse y recuperar la posesión para reincorporarse al juego.

\section{Modificaciones estructurales para el diseño de juegos}

En este apartado daremos algunas pistas al objeto de potenciar la creación de actividades lúdicas nuevas y significativas que pretendan garantizar la seguridad, reducir el riesgo de lesión, y aumentar la participación de los alumnos, con independencia de su condición y competencia motriz.

1. Móviles. Los balones de baloncesto, fútbol o incluso los clásicos de voleibol no resultan apropiados. Una de las claves en la construcción de pelotas con materiales de desecho es no prensar demasiado la materia prima escogida (papel de periódico, bolsas) y no tensar en exceso la cinta de embalar. Las posibilidades son múltiples: peluches, pompones, pelotas con velcro, pelotas de esponja, indiacas con bolsas de plástico, discos voladores de trapo o de cartón acolchado con plásticos de burbujas..., en definitiva, todo aquello que reduzca el impacto. El tamaño es importante, una pelota pequeña hace que el juego sea más desafiante, mientras que una mayor lo facilita. 
2. Implemento. Usar utensilios construidos, en lugar de convencionales, abre una puerta a la creación de múltiples propuestas. Por ejemplo, en vez de utilizar el stick del rock-it-ball (una especie crosse con una cesta en cada extremo del palo), se pueden emplear otros objetos de golpeo y lanzamiento como palas, cestas simples o raquetas (Méndez, 2003). Un ejemplo lo encontramos en la adaptación de pies quietos con cesta guante (fig. 7).

3. Metas. Puesto que éste es el foco de la controversia, son necesarias restricciones para salvaguardar la seguridad. En primer lugar, habrá que clarificar qué parte del cuerpo se considera diana (por debajo de la línea delos hombros, de la cintura, de las rodillas). En pepperches(Luxemburgo), los jugadores tras la huída colocan los brazos en círculo por encima de la cabeza para dibujar una diana. En segundo lugar, se puede sustituir el cuerpo de la liebre como blanco por algún objeto que éste porta, por ejemplo, equipando a cada jugador con recipientes en donde los contrincantes tratan de introducir móviles. Este es el caso del juego de «mochileros» para el que se construyen «mochilas» con garrafas de agua (fig. 8). Otra opción es emplear una sábana con agujeros de diferente tamaño, que es portada y estirada por una pareja que huye. El resto trata de hacer diana obteniendo la mayor puntuación posible. densidadalta dealumnos se deberáreducirla velocidad del juego limitando la persecución a caminar o marchar para evitar choques.

5. Jugadores (compañeros/adversarios). Para evitar que haya niños que eludan el juego o aumentar el tiempo de participación se debe reducir el número de jugadores por bando o juego. Los equipos deben estar bien nivelados en cuanto a fuerza, y equilibrar el número de cazadores y de liebres para aumentar la participación y el requerimiento físico. No parece razonable contar solo con un perseguidor de un gran grupo, puesto que el nivel de actividad de los segundos será mínimo mientras que el cazador acabará extenuando.

6. Tiempo. Cada cierto tiempo se deben rotar los roles para garantizar una participación suficiente y compensada.

7. Reglamento. Presentamos algunas modificaciones de las reglas, que se sumarán a los esfuerzos por sensibilizar a los niños del riesgo de los juegos de lanzamiento a dianas humanas cuando se emplean materiales duros.

$\varnothing \quad$ Lanzar la pelota rodándola por el suelo y rotar los roles de lanzador y el que esquiva cada poco tiempo (Jackson, 2001)

$\varnothing \quad$ Promover lanzamientos por debajo de la cadera para reducir la potencia.

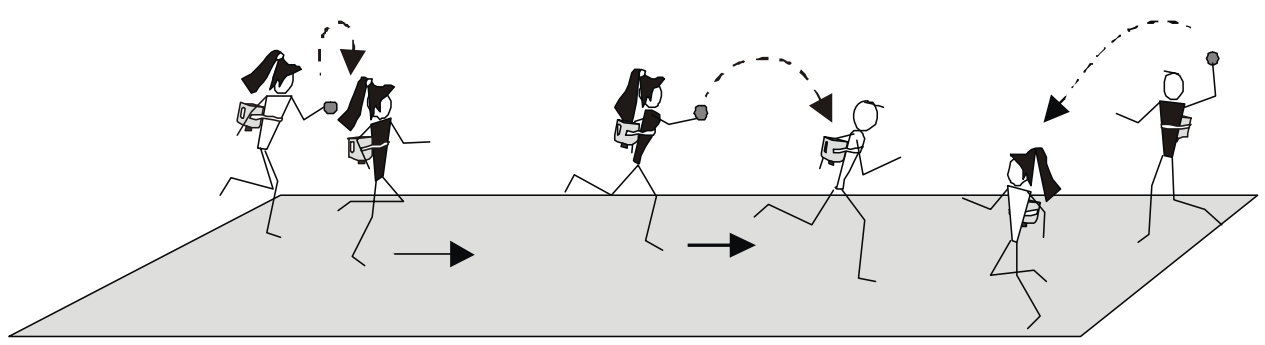

$\varnothing \quad$ Considerar que la pelota deba botar en el suelo antes de impactar en el jugador.

$\varnothing \quad$ Penalizar los impactos en la cabeza o excesivamente fuertes.

$\varnothing \quad$ Frentealareglade la eliminación definitiva, contemplar reglas de reincorporación rápida al juego tras cumplir determinadas acciones: que

Fig. 8. Mochileros (Méndez, 2003)

En tercer lugar, se pueden construir petos (a los que se adhiere una cara del velcro) y pelotas de papel (con la otra parte del velcro). En EEUU ya existe esta equipación comercializada para jugar a tagball, lo que constituye un vivero de múltiples juegos.

Finalmente, se puede profundizar en la idea de objetos móviles, como en el tesoro movedizo (Méndez y Méndez, 1996) donde cuatro equipos disparan desde varios flancos para aproximar un balón medicinal a cualquiera de las otras líneas (fig. 9).

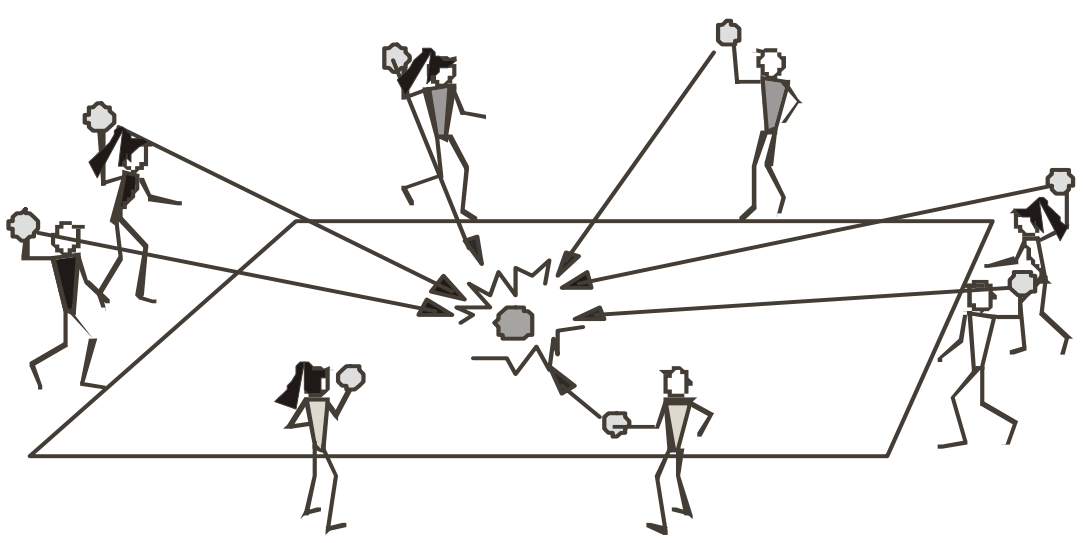

el balón toque al que estásentado esperando, que el jugador «congelado» toque a un adversario que pasa cerca...

En definitiva, reducir las posibilidades de humillación pasa por conseguir equilibrar las fuerzas entre los rivales, restringir las conductas violentas y generar un clima de respeto.

8. Habilidades deportivas. Variar las habilidades a realizar: andar, marchar, correr, saltar. . . lanzar rodando, lanzar por el aire, botar, chutar, golpear con la mano o con objetos un móvil. .

9. Pensamiento táctico. Navarro (2006) propone transformar los juegos duales (dos equipos) en tríadas (tres equipos enfrentados); el resultado es un aumento del requerimiento perceptivo y de la complejidad táctica de los juegos.

Finalmente, deseamos concluir que metodológicamente es preciso delimitar los objetivos de cada propuesta para no saturar al alumnado. Cuando el propósito del juego sea trabajar la condición física, no parece lógico plantear simultáneamente retos tácticos nuevos, puesto que la fatiga acumulada reduce las posibilidades de respuestas reflexivas (Belka, 1998).

\section{Referencias bibliográficas}

Almond, L. (1986). Reflecting on themes: a games classification. En Thorpe, D., Bunker, D., y Almond, L. (Eds). Rethinking Games Teaching. Loughborough: Loughborough

Fig. 9. Tesoro movedizo

4. Terreno de juego. Las dimensiones del terreno de juego serán acordes con las características del alumnado. Amayor distancia, mayor dificultad de conseguir la cacería. En espacios reducidos o con una
University, 71 - 72

Belka, D. E. (1998). «Strategies for Teaching Tag Games», Journal of Physical Education, Recreation and Dance, 69 (8), 40-43.

Belka, D. E. (2006). «What do tag games teach?», Teaching Elementary Physical Education, 17(3), 35-36. 
Casey, A., Dyson B. y Campbell A. (2009). Action research in physical education: focusing beyond myself through cooperative learning. Educational Action Research, Vol. 17, 3, 407-423

Deutsch, J. (2007). From Hall of Shame to Hall of Fame: Transforming traditional elementary activities, MJOHPERD, 17, 48-52.

González Herrero, E. (1996). El aprendizaje de los juegos deportivos. En Moreno Murcia, J. A. y Rodríguez García, P. L. (Coord.). Aprendizaje deportivo. Murcia: Universidad de Murcia, 185- 204.

Griffin, L.L. y Butler, J.I. (2005). Teaching Games for Understanding: Theory, research, and practice. Champaign, IL: Human Kinetics.

Griffin, L.L., Mitchell, S.A. y Oslin, J.L. (1997). Teaching Sport Concepts and Skillls. Atactical Games Approach. Champaign: IL. Human Kinetics.

Hanrahan, S.J. y Carlson, T.B. (2000). Games Skills. A Fun Approach to Learning Sport Skills. Champaign, IL: Human Kinetics.

Hernández, J. (1994). Fundamentos del deporte. Análisis de las es-tructuras del juego deportivo. Barcelona: INDE.

Jackson, S.L. (2001). «Dodgeball vs. Dodge Ball activities». Sportapolis.

Maurer, M. R. (2006). «What if dodgeball had it own Nacional Standard». Teaching Elementary Physical Education, 17(1), 6-7.

McDonald, J. (2005). Dodging «Dodgeball». Physical and Health Education, 18-19.

Méndez Giménez, A. (2001). «El diseño de juegos modificados: Un marco de encuentro entre la variabilidad estructural y la intencionalidad educativa», Tándem. Didáctica de la Educación Física, 3, 110-122.

Méndez Giménez, A. (2003). Nuevas propuestas lúdicas para el desarrollo curricular de Educación Física. Barcelona: Paidrotribo.

Méndez Giménez, A. (2005). «Una iniciación deportiva de calidad con materiales autoconstruidos. El ejemplo del ringo en el marco de un modelo comprensivo-estructural». Tándem. Didáctica de la Educación Física, 18, 61-69.
Méndez Giménez, A. (2006a). «Los juegos de diana desde un modelo comprensivo-estructural basado en la auto-construcción de materiales: el boomerang enla educación física». Tándem. Didáctica de la Educación Física. 20,101-111.

Méndez Giménez, A. (2006b). «El ultimate con materiales de desecho desde un enfoque comprensivo estructural. Tándem. Didáctica de la Educación Física, 21, 102-117.

Méndez Giménez, A. (2009a). «La enseñanza de los juegos de golpeo y fildeo en el contexto escolar a partir dematerial reciclado». Tándem. Didáctica de la Educación Física, 29, 105-118.

Méndez Giménez, A (2009b) (coord.). Modelos actuales de iniciación deportiva escolar. Unidades didácticas sobre deportes de invasión. Sevilla: Ed.Wanceulen.

Méndez Giménez, A. y Méndez Giménez, C. (1996). Los juegos en el currículum de la Educación Física. Más de 1000 juegos para el desarrollo motor. Barcelona. Ed: Pai-dotribo.

Mitchell, S.A., Oslin, J.L. y Griffin, L.L. (2003). Sport Foundations for Elementary Physical Education. Champaign, Il.: Human Kinetics.

Navarro Adelantado, V. (2006). Del juego al deporte. Discusión estructural, antropológica y pedagógica», en Juego y deporte en el ámbito escolar: aspectos curriculares y actuaciones prácticas. Madrid: MEC-Instituto Superior de Formación del Profesorado. Pp. 67-107.

Thorpe, R., Bunker, D. y Almond, L. (1986). Rethinking Games Teaching. Lough-borough. UK. University of Technology. Department of Physical Education and Sport Science.

Townsend, J. S., Mohr, D. J., Waronsky C. C. y Grana, M. M. (2006). «Non-Elimination Tag: A Multidisciplinary Approach». Teaching Elementary Physical Education, 17(1), 35-36.

Williams, N. (1992). The physical education hall of shame. Journal of Physical Education, Recreation and Dance, 63 (6), 57-60.

Williams, N. (1994). The physical education hall of shame, part II. Journal of Physical Education, Recreation and Dance, 65 (2), 1720.

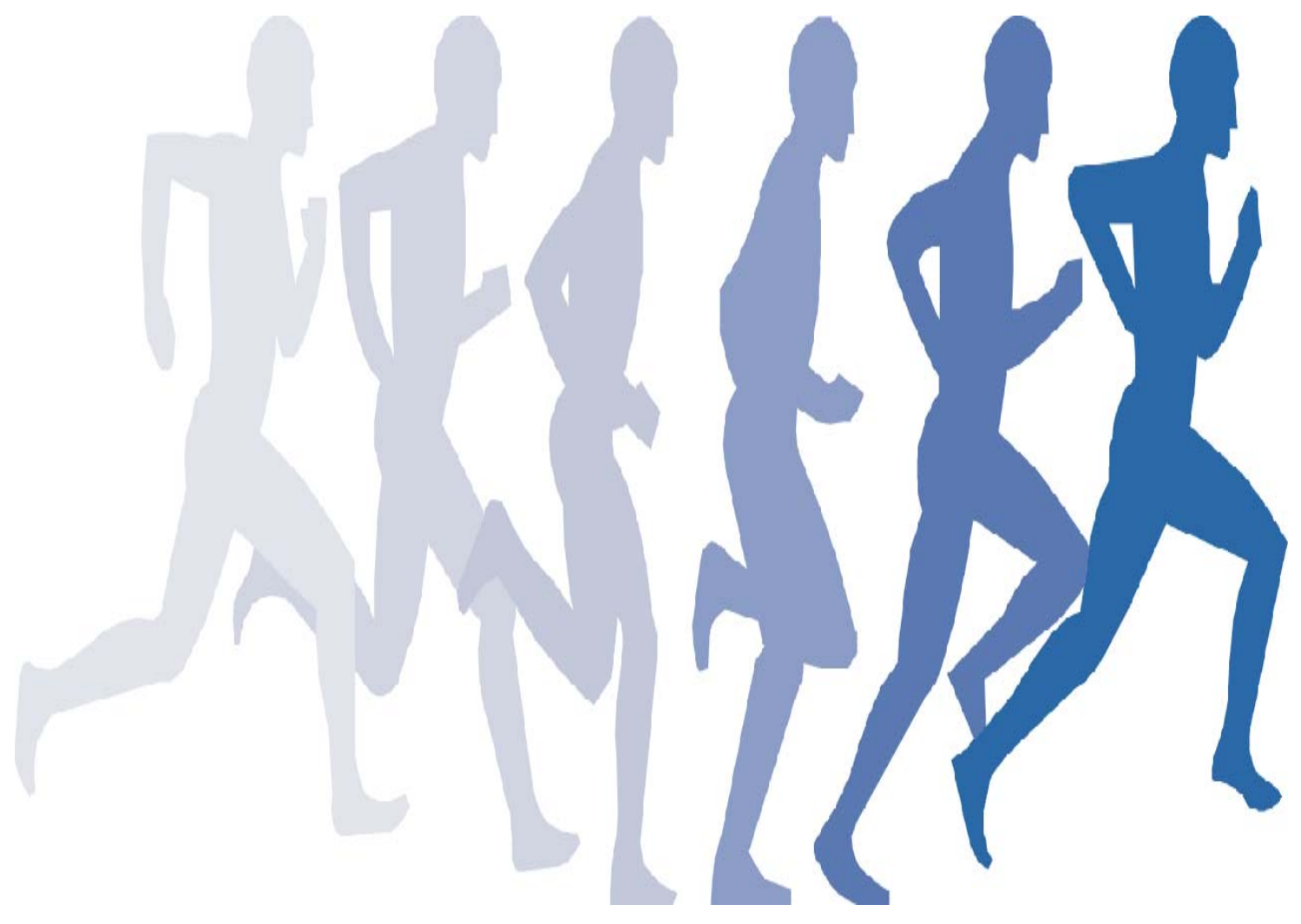

